\title{
Diffraction of water waves by a submerged vertical plate in uniform finite depth water
}

\author{
B. C. Das \\ Department of Basic Science and Humanities, University of Engineering and Management, Kolkata-160, India
}

\begin{abstract}
This paper involves oblique scattering of surface water waves by a bottom standing thin vertical barrier present in uniform finite depth water. Use of Havelock's expansion of water wave potential reduces each problem to two integral equations of the first kind, one on horizontal component of velocity across the gap and the other on the difference of potentials across the barrier. Single-term Galerkin technique involving constants as basis functions multiplied by appropriate weight functions whose forms are dictated by the edge conditions is employed to find approximate solutions of the two integral equations. This is in contrast to somewhat complicated known functions used earlier in the literature as basis. Very close upper and lower bounds of the reflection and transmission coefficients for each configuration are evaluated numerically for various values of the parameters. Their averages produce very good numerical estimates for these coefficients and these are depicted graphically against the wavenumber and the incident angle for each barrier.
\end{abstract}

PACS numbers:

Key words: Oblique scattering, linear theory, first kind integral equations, finite depth, single-term Galerkin approximation, reflection and transmission coefficients.

\section{Introduction}

Water wave scattering by obstacles involving floating and submerged bodies of various geometrical shapes form an extremely important class of problems in the linearized theory of water waves. When a train of surface water waves is incidence on an obstacle then it is partially transmitted and partially reflected by the bodies. Evaluation of the reflection and transmission coefficients is the most important task for these problems.

Earlier, Dean [1] found the explicit solution for the problem of water wave scattering by a submerged thin vertical barrier extending infinitely downwards in deep water. He used the theory of complex variables to solve this problem. Shortly afterwards Ursell [2] used Havelock's [3] expansion of water wave potential to solve the complementary problem of Dean [1] i.e, water wave scattering by a partially immersed thin vertical barrier in deep water. Williams [4] also solved the partially immersed barrier problem by using reduction technique. Evans [5] solved the problem of water wave scattering by a submersed vertical plate. He used the theory of complex analysis leading to solving a Riemann-Hilbert problem.

Water wave scattering problems involving bottom standing thin vertical barriers present in uniform finite depth water do not possess explicit solutions. However, a number of approximate methods have been used to solve these problems in the sense that the reflection and transmission coefficients were obtained approximately. For normal incidence, these finite depth problems were considered by Goswami [6-8] who used an integral equation formulation based on Green's integral theorem. In each case, a perturbation analysis about the explicit deep water solution was employed by assuming the depth of water to be large but finite. He concluded that the effect of finite depth on the reflection and transmission coefficients are algebraic rather than exponential for large but finite depth of water. However, the method is not suitable for moderate depth of water. The surface piercing barrier problem for finite depth and normal incidence has been studied by Smith [9] who used eigenfunction expansions for the velocity potential. He transformed his problem to an infinite system of linear equations. But convergence of the solution of the infinite system is very slow and thus the method is not very efficient computationally. Later Losada et. al [10] used eigenfunction expansions for the velocity potential and reduced the problem to an infinite system of equations by using the principle of least square. Here also convergence of the method is very slow and as such is not very attractive computationally. Also, Mandal and Dolai [11] used single-term Galerkin technique involving the explicit solutions of the corresponding integral equations for the case of normal incidence and deep water given by Ursell [2]. However, these single terms are somewhat complicated. Here we also used single-term Galerkin method in the mathematical analysis but the single terms are simply constants multiplied by appropriate weight functions, the constants being taken to be unity and the forms of the weight functions being dictated by the conditions of the horizontal component of velocity and difference of potential functions at the edge of a barrier. By using this method very accurate numerical estimates for the reflection and transmission coefficients are obtained. This method appears to be very simple and straightforward in comparison to the methods employed by Goswami [6-8], Smith [9], Losada et. al [10] and Mandal and Dolai [11].

\section{Mathematical formulation of the problem:}

A train of surface water waves are obliquely incident

\section{Email:findbablu10@gmail.com}


on a thin vertical barrier present in uniform finite depth water $x=0, y \in L$ from the direction of negative infinity. Here $L=(b, h)$ for the bottom standing submerged barrier, the $y$-axis being chosen vertically downwards into the fluid region through the barrier, the $(x, z)$-plane is the position of the undisturbed free surface, $h$ is the uniform depth of the water. The incident wave train is described by the velocity potential $R e\left\{\phi^{i n c}(x, y) e^{i \nu z-i \sigma t}\right\}$ where

$$
\phi^{i n c}(x, y)=\frac{\cosh k_{0}(h-y)}{\cosh k_{0} h} e^{i \mu x} .
$$

In $(2.1), k_{0}$ is the unique positive root of the transcendental equation

$$
k \tanh k h=K
$$

where $K=\frac{\sigma^{2}}{g}, g$ being the acceleration due to gravity and $\mu=k_{0} \cos \alpha, \nu=k_{0} \sin \alpha, \alpha$ being the angle of incidence of the wave train. Due to the geometrical symmetry, $z$ can be eliminated by assuming the velocity potential of the resulting motion in the form $\operatorname{Re}\left\{\phi(x, y) e^{i \nu z-i \sigma t}\right\}$. Then $\phi(x, y)$ satisfies the modified Helmholtz equation with parameter $\nu$ in the fluid region

$$
\left(\nabla^{2}-\nu^{2}\right) \phi=0,0 \leq y \leq h,
$$

the free surface condition

$$
K \phi+\frac{\partial \phi}{\partial y}=0, \text { on } y=0
$$

the condition on the barrier

$$
\frac{\partial \phi}{\partial x}=0, \text { on } x=0, y \in L,
$$

the edge condition

$$
r^{\frac{1}{2}} \nabla \phi \text { is bounded as } r \longrightarrow 0
$$

where $r$ is the distance from a submerged edge of the barrier,

the bottom condition

$$
\frac{\partial \phi}{\partial y}=0 \text { on } y=h
$$

And the condition as $|x| \rightarrow \infty$ given by

$$
\phi(x, y) \rightarrow\left\{\begin{array}{l}
T \phi^{i n c}(x, y) \text { as } x \rightarrow \infty \\
\phi^{i n c}(x, y)+R \phi^{i n c}(-x, y) \text { as } x \rightarrow-\infty
\end{array}\right.
$$

where $T$ and $R$ denote the transmission and reflection coefficients respectively.

\section{Method of solution}

Using Havelock's [3] expansion of water wave potential, solution of $\phi(x, y)$ satisfying the equation (2.3) and the conditions $(2.4),(2.7)$, and (2.8) is expressed as

$$
\phi(x, y)=
$$

$$
\left\{\begin{array}{l}
T \phi^{i n c}(x, y)+\sum_{n=1}^{\infty} A_{n} \cos k_{n}(h-y) e^{-s_{n} x}, x>0, \\
\phi^{i n c}(x, y)+R \phi^{i n c}(-x, y)+\sum_{n=1}^{\infty} B_{n} \cos k_{n}(h-y) e^{s_{n} x}, x<0
\end{array}\right.
$$

where $s_{n}=\left(k_{n}^{2}+\nu^{2}\right)^{\frac{1}{2}}, \nu=k_{0} \sin \alpha$ and $k_{n}(n=$ $1,2,3, \ldots)$ are the positive real roots of

$$
K+k \tan k h=0,
$$

and the $A_{n}$ 's and $B_{n}$ 's are to be determined.

Let $p(y)$ and $q(y)$ denoted the horizontal component of velocity and difference of velocity potential respectively across the plane of the barrier, then

$$
p(y)=0 \text { on } y \in L
$$

and

$$
q(y)=0 \text { for } y \in \bar{L}=(0, h)-L .
$$

Also due to the edge condition (2.6), $p(y), q(y)$ must have the behaviours

$$
\begin{gathered}
p(y)=O\left(|y-c|^{-1 / 2}\right) \text { as } y \rightarrow c, \\
q(y)=O\left(|y-c|^{1 / 2}\right) \text { as } y \rightarrow c,
\end{gathered}
$$

where $c=a$ for $L=L_{1}$ and $c=b$ for $L=L_{2}$.

Using the representations (3.1), we find that

$$
\begin{gathered}
p(y)=\frac{\partial \phi}{\partial x}(+0, y)=\frac{i \mu T \cosh k_{0}(h-y)}{\cosh k_{0} h}-\sum_{n=1}^{\infty} s_{n} A_{n} \cos k_{n}(h-y) \\
=\frac{\partial \phi}{\partial x}(-0, y)= \\
\frac{i \mu(1-R) \cosh k_{0}(h-y)}{\cosh k_{0} h}+\sum_{n=1}^{\infty} s_{n} B_{n} \cos k_{n}(h-y), 0<y<h,
\end{gathered}
$$

and

$$
\begin{gathered}
q(y)=\phi(+0, y)-\phi(-0, y) \\
=(T-R-1) \frac{\cos k_{0}(h-y)}{\cosh k_{0} h}+\sum_{n=1}^{\infty} s_{n}\left(A_{n}-B_{n}\right) \cos k_{n}(h-y), 0<y<h .
\end{gathered}
$$

By using Havelock's [3] inversion formula we find from the relations (3.7) and (3.8) after using (3.3) and (3.4) that

$T=1-R=-\frac{4 i k_{0} \cosh k_{0} h}{\mu\left(2 k_{0} h+\sinh ^{2} k_{0} h\right)} \int_{\bar{L}} p(y) \cosh k_{0}(h-y) d y$, 


$$
\begin{gathered}
A_{n}=-B_{n}=-\frac{4 k_{n}}{s_{n}\left(2 k_{n} h+\sin 2 k_{n} h\right)} \int_{\bar{L}} p(y) \cosh k_{n}(h-y) d y \\
R=1-R=-\frac{2 k_{0} \cosh k_{0} h}{2 k_{0} h+\sinh ^{2} k_{0} h} \int_{L} q(y) \cosh k_{0}(h-y) d y,
\end{gathered}
$$

and

$$
A_{n}=-\frac{2 k_{n}}{\left(2 k_{n} h+\sin 2 k_{n} h\right)} \int_{L} q(y) \cosh k_{n}(h-y) d y .
$$

An integral equation for $q(y), y \in L$, is obtained by using the equation (3.3) for $p(y)$ in the second of the relations (3.7) after $A_{n}$ is substituted from the relation (3.12). Thus we find

$$
\begin{aligned}
& \frac{\cosh ^{2} k_{0} h}{2 k_{0} h+\sinh 2 k_{0} h} \int_{L} q(u) \mathcal{M}(y, u) d u \\
& =\frac{i \mu}{2}(1-R) \frac{\cosh k_{0}(h-y)}{\cosh k_{0} h}, y \in L,
\end{aligned}
$$

where

$$
\mathcal{M}(y, u)=
$$

$\frac{2 k_{0} h+\sinh 2 k_{0} h}{\cosh ^{2} k_{0} h} \lim _{\epsilon \rightarrow+0} \sum_{n=1}^{\infty} \frac{s_{n} k_{n} \cos k_{n}(h-y) \cos k_{n}(h-u)}{2 k_{n} h+\sin 2 k_{n} h} e^{-\epsilon k_{n}}$

the exponential term being introduced to ensure the convergence of the series.

Similarly, an integral equation for $p(y)$ is obtained as

$\frac{\cosh ^{2} k_{0} h}{2 k_{0} h+\sinh 2 k_{0} h} \int_{\bar{L}} p(u) \mathcal{N}(y, u) d u=-\frac{R}{4} \frac{\cosh k_{0}(h-y)}{\cosh k_{0} h}, y \in \bar{L}$,

where

$$
\begin{gathered}
\mathcal{N}(y, u)= \\
\frac{2 k_{0} h+\sinh 2 k_{0} h}{\cosh ^{2} k_{0} h} \lim _{\epsilon \rightarrow+0} \sum_{n=1}^{\infty} \frac{k_{n} \cos k_{n}(h-y) \cos k_{n}(h-u)}{s_{n}\left(2 k_{n} h+\sin 2 k_{n} h\right)} e^{-\epsilon k_{n}}
\end{gathered}
$$

where $y, u \in \bar{L}$, the exponential term also being introduced here to ensure convergence of the series. If we define

$$
G(y)=\frac{2 \cosh ^{2} k_{0} h}{i \mu(1-R)\left(2 k_{0} h+\sinh 2 k_{0} h\right)} q(y), y \in L,
$$

and

$$
F(y)=-\frac{4 \cosh ^{2} k_{0} h}{R\left(2 k_{0} h+\sinh 2 k_{0} h\right)} p(y), y \in \bar{L},
$$

then we find that $G(y)$ and $(F(y))$ satisfy the integral equations

$$
\int_{L} G(y) \mathcal{M}(y, u) d u=\frac{\cosh k_{0}(h-y)}{\cosh k_{0} h}, y \in L,
$$

and

$$
\int_{\bar{L}} F(y) \mathcal{N}(y, u) d u=\frac{\cosh k_{0}(h-y)}{\cosh k_{0} h}, y \in \bar{L} .
$$

Again, using the relations (3.17) and (3.18) in the equations (3.11) and (3.9) respectively, we define

$$
\int_{L} G(y) \frac{\cosh k_{0}(h-y)}{\cosh k_{0} h} d y=\frac{1}{k_{0}^{2} C},
$$

and

$$
\begin{gathered}
\int_{\bar{L}} F(y) \frac{\cosh k_{0}(h-y)}{\cosh k_{0} h} d y=C, \\
C=\frac{1-R}{i R} \cos \alpha .
\end{gathered}
$$

It may be noted that $C, F(y)$ and $G(y)$ are real valued equations which follows by examining the equations (3.19) to $(3.23)$.

\section{Upper and Lower Bounds for $C$}

Following Evans and Morris [12], we define an inner product

$$
<f, g>=\int_{L} f(y) g(y) d y .
$$

Then obviously $<f(y), g(y)>$ is symmetric and linear. Also the operator $\mathcal{M}$ defined by

$$
(\mathcal{M g})(y)=<\mathcal{M}(y, u), g(u)>
$$

,is linear, self-adjoint and positive semi-definite.

We choose a single-term Galerkin approximation for the solution of the integral equation (3.19) in the form

$$
G(y) \approx a_{0} q_{0}(y), y \in L
$$

where $a_{0}$ is a constant and $q_{0}(y)$ is to be chosen suitably. Then

$$
a_{0}=\frac{<q_{0}(y), \frac{\cosh k_{0}(h-y)}{\cosh k_{0} h}>}{<q_{0}(y),\left(\mathcal{M} q_{0}\right)(y)>} .
$$

Hence, using the approximation (4.3) for $G(y)$ in the relation (3.21) and using the same argument as in the Evans and Morris [12], we find

$\frac{1}{k_{0} C}=<G(y), \frac{\cosh k_{0}(h-y)}{\cosh k_{0} h}>\geq<a_{0} q_{0}(y), \frac{\cosh k_{0}(h-y)}{\cosh k_{0} h}>$.

Thus we find

$$
C \leq A
$$

where

$A=\frac{\frac{2 k_{0} h+\sinh 2 k_{0} h}{\cosh ^{2} k_{0} h} \sum_{n=1}^{\infty} \frac{s_{n} k_{n}}{2 k_{n} h+\sin 2 k_{n} h}\left[\int_{L} q_{0}(y) \cos k_{n}(h-y) d y\right]^{2}}{k_{0}^{2}\left[\int_{L} q_{0}(y) \frac{\cosh k_{0}(h-y)}{\cosh k_{0} h} d y\right]^{2}}$

Thus $A$ can be regarded as an upper bound of the unknown constant $C$.

Again, if we define another inner product by

$$
<<f, g>>=\int_{\bar{L}} f(y) g(y) d y
$$

and another operator $\mathcal{N}$ by

$$
(\mathcal{N} f)(y)=<<\mathcal{N}(y, u), f(u)>>,
$$

then it is obvious that $\langle<f, g\rangle>$ is linear, symmetric and also the operator $\mathcal{N}$ is linear, self-adjoint and positive semidefinite. 
For the solution of the integral equation (3.20), we choose a one-term Galerkin approximation as

$$
F(y) \approx b_{0} p_{0}(y), y \in \bar{L},
$$

where $b_{0}$ is an unknown constant and $p_{0}(y)$ is to be chosen suitable. Then $b_{0}$ is found to be

$$
b_{0}=\frac{<<p_{0}(y), \frac{\cosh k_{0}(h-y)}{\cosh k_{0} h}>>}{<<p_{0}(y),\left(\mathcal{N} p_{0}\right)(y)>>} .
$$

Hence, using the approximation (4.10) for $F(y)$ in the relation (3.22) and using the same argument as in the Evans and Morris [12], we find that

$$
\begin{gathered}
C= \\
<<F(y), \frac{\cosh k_{0}(h-y)}{\cosh k_{0} h}>>\leq<<b_{0} p_{0}(y), \frac{\cosh k_{0}(h-y)}{\cosh k_{0} h}>>.
\end{gathered}
$$

Thus we find

$$
C \geq B
$$

where

$$
\begin{gathered}
B= \\
\frac{\left[\int_{\bar{L}} p_{0}(y) \frac{\cosh k_{0}(h-y)}{\cosh k_{0} h} d y\right]^{2}}{\frac{2 k_{0} h+\sinh 2 k_{0} h}{\cosh ^{2} k_{0} h} \sum_{n=1}^{\infty} \frac{k_{n}}{s_{n}\left(2 k_{n} h+\sin 2 k_{n} h\right)}\left[\int_{\bar{L}} p_{0}(y) \cos k_{n}(h-y) d y\right]^{2}}
\end{gathered}
$$

Thus upper and lower bounds for the unknown real constant $C$ defined by the relation (3.23) are obtained as $A$ and $B$ respectively. Hence for the unknown real constant $C$, we find

$$
B \leq C \leq A \text {. }
$$

Hence upper and lower bound for $|R|$ and $|T|(=|1-R|)$ are obtained as

$$
R_{1} \leq|R| \leq R_{2}, T_{1} \leq|T| \leq T_{2}
$$

where

$$
\begin{aligned}
& R_{1}=\frac{\cos \alpha}{\left(A^{2}+\cos ^{2} \alpha\right)^{\frac{1}{2}}}, R_{2}=\frac{\cos \alpha}{\left(B^{2}+\cos ^{2} \alpha\right)^{\frac{1}{2}}}, \\
& T_{1}=\frac{B}{\left(A^{2}+\cos ^{2} \alpha\right)^{\frac{1}{2}}}, T_{2}=\frac{A}{\left(B^{2}+\cos ^{2} \alpha\right)^{\frac{1}{2}}} .
\end{aligned}
$$

In the next sections bottom standing submerged barrier is considered, upper and lower bounds for the reflection and transmission coefficients are evaluated in each case for the various values of the different parameters involved.

\section{Submerged barrier extending down to the bottom}

Here $L=(b, h)$ so that $\bar{L}=(0, b)$. This problem was considered by Mandal and Dolai [11] and Losada et. al [10]. However, Mandal and Dolai [11] used one-term Galerkin approximation using Ursell's [2] explicit solution for deep water and normal incidence while Losada et. al [10] used the principle of least square to obtain the reflection and transmission coefficients numerically. Here we use one-term Galerkin approximations involving constants multiplied by appropriate weight functions. For $q_{0}(y)$ and $p_{0}(y)$ we choose

$$
q_{0}(y)=\left(\left(\frac{y}{b}\right)^{2}-1\right)^{\frac{1}{2}}, b<y<h
$$

and

$$
p_{0}(y)=\left(1-\left(\frac{y}{b}\right)^{2}\right)^{-\frac{1}{2}}, 0<y<b
$$

taking the constants as unity as these do not affect $A$ and $B$ given by (4.7) and (4.14) respectively. These are substituted in the equations (4.7) and (4.14) to find the values of $A$ and $B$ and we get

$$
\begin{gathered}
A= \\
\frac{\frac{2 k_{0} h+\sinh 2 k_{0} h}{\cosh ^{2} k_{0} h} \sum_{n=1}^{\infty} \frac{s_{n} k_{n}}{2 k_{n} h+\sin 2 k_{n} h}\left[\int_{b}^{h} q_{0}(y) \cos k_{n}(h-y) d y\right]^{2}}{k_{0}^{2}\left[\int_{b}^{h} q_{0}(y) \frac{\cosh k_{0}(h-y)}{\cosh k_{0} h} d y\right]^{2}}
\end{gathered}
$$

and

$$
\begin{gathered}
B= \\
\frac{\left[\int_{0}^{b} p_{0}(y) \frac{\cosh k_{0}(h-y)}{\cosh k_{0} h} d y\right]^{2}}{\frac{2 k_{0} h+\sinh 2 k_{0} h}{\cosh ^{2} k_{0} h} \sum_{n=1}^{\infty} \frac{k_{n}}{s_{n}\left(2 k_{n} h+\sin 2 k_{n} h\right)}\left[\int_{0}^{b} p_{0}(y) \cos k_{n}(h-y) d y\right]^{2}} .
\end{gathered}
$$

The lower and upper bounds for the reflection and transmission coefficients are now calculated by using the relations (4.17) and (4.18) (taking $L=(b, h)$ ) for various

\begin{tabular}{|c|c|c|c|c|c|c|}
\hline & $\alpha=0^{0}$ & & $\alpha=30^{0}$ & & $\alpha=60^{0}$ & \\
\hline$b / h$ & $R_{1}$ & $R_{2}$ & $R_{1}$ & $R_{2}$ & $R_{1}$ & $R_{2}$ \\
\hline 0.2 & 0.291453 & 0.292491 & 0.255010 & 0.255545 & 0.150066 & 0.150234 \\
\hline 0.4 & 0.139607 & 0.139685 & 0.120547 & 0.121000 & 0.069814 & 0.069895 \\
\hline 0.6 & 0.057339 & 0.0573464 & 0.049533 & 0.0496181 & 0.028515 & 0.028595 \\
\hline 0.8 & 0.015263 & 0.0155993 & 0.013471 & 0.0135015 & 0.007772 & 0.007786 \\
\hline
\end{tabular}
values of different parameters and the angle of incidence $\alpha$.

\section{Numerical results}

From the Table 1 , it is seen that $R_{1}$ and $R_{2}$ coincide in most cases upto 2 to 4 decimal places and hence their averages provide very good accurate numerical estimates for the reflection coefficient. Similar results have been obtained for $T_{1}$ and $T_{2}$ and their averages provide very accurate numerical estimates for the transmission coefficient $|T|$. However these results are not given here in a tabular form. It has been checked that these numerical estimates satisfy the energy identity $|R|^{2}+|T|^{2}=1$, which provides a partial check on the correctness of the method. There are also other checks as described below.

Table 1. Lower and upper bounds for the reflection coefficient $|R|$ for $K h=0.2$ 


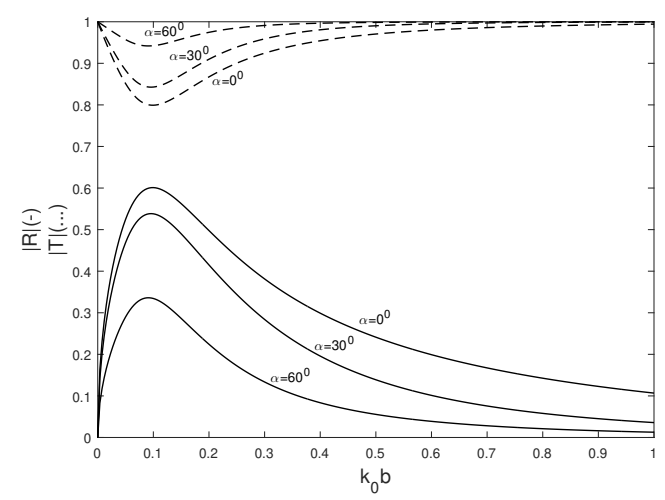

FIG. 1: $|R|,|T|$ vs $k_{0} b$ for different values of $\alpha$ and $b / h=0.1$

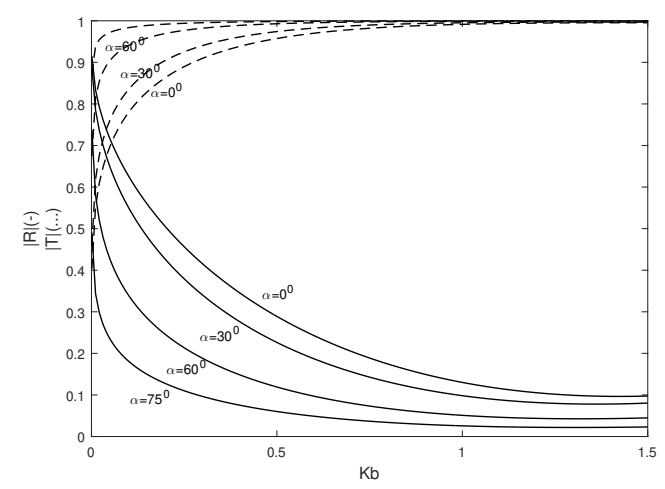

FIG. 2: $|R|,|T|$ vs $K b$ for different values of $\alpha$ and $b / h=0.01$

In figure $1,|R|$ and $|T|$ are depicted against the wavenumber $k_{0} b$ for different values of $\alpha$ and $a / h=0.1$. It is observed from this figure that for $\alpha=0^{0}$, the curves of $|R|$ and $|T|$ coincide with the curves of $|R|$ and $|T|$ in the figure 3 of Porter and Evans [13] corresponding to $b / h=0.1$. From this figure, it is seen that for fixed $b / h=0.1$ and $\alpha$, the reflection coefficient $|R|$ first increases and the transmission coefficient $|T|$ first decreases as $k_{0} b$ increases and then $|R|$ decreases and $|T|$ increases for further increase of $k_{0} b$. This is plausible since for low wavenumbers, the wave potential behaves like a horizontal flow far from the barrier so that most of the wave energy is transmitted through the upper part of the submerged barrier and for large $k_{0} b$, most of the wave energy is confined near the free surface so that a large proportion of the wave energy is transmitted. It may be noted that $|R|$ and $|T|$ decreases and increases respectively monotonically as angle of incidence increases. In particular, for the grazing incidence $\left(\alpha \approx \frac{\pi}{2}\right),|R|$ and $|T|$ asymptotically becomes zero and unity respectively which are expected from physical consideration. This will also be apparent from figure 3 in which $|R|$ and $|T|$ are depicted against $\alpha$ for $b / h=0.1$ and different $k_{0} b$.

In figure 2, $|R|$ and $|T|$ are depicted against wavenumber $K b$ for $b / h=0.01$ and for different $\alpha$. For $\alpha=0^{0}$, the curves of $|R|$ and $|T|$ almost coincide with the curves given by Dean [1] for the case of a thin vertical barrier submerged

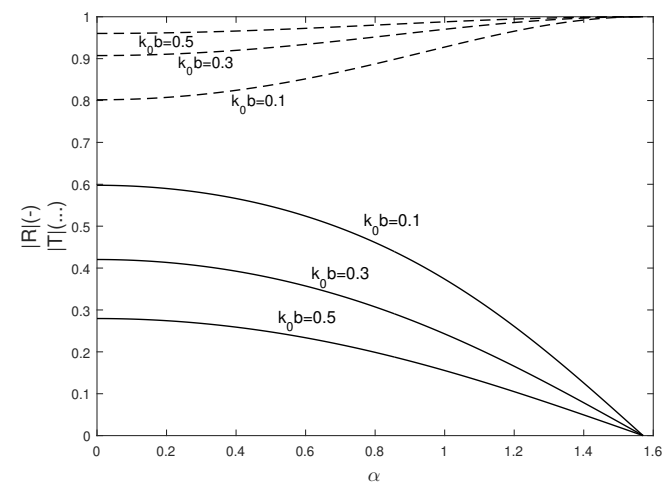

FIG. 3: $|R|,|T|$ vs $\alpha$ for different values of $k_{0} b$ and $b / h=0.1$

in deep water. This is due to the fact that as the water depth is hundred times the depth of the upper edge of the barrier below the mean free surface, the water can be assumed to be deep. It is seen that for fixed angle of incidence $\alpha$ as the wavenumber $K b$ increases $|R|$ decreases while $|T|$ increases. This observation is in contrast with the observation in figure 1 wherein, regarded as function of the wave number $k_{0} b,|R|$ has a maximum and $|T|$ has a minimum for a particular value of $k_{0} b$ for each angle of incidence. As mentioned by Porter and Evans [13], this is due to the fact that total reflection $(|R| \rightarrow 0)$ occurs when waves of large wavelengths $\left(k_{0} b \rightarrow 0\right)$ are incident on a very long submerged barriers $(b / h=0.01)$. The effect of $|R|$ and $|T|$ on the angle of incidence $\alpha$ (in radian) is shown in figure 3 for $b / h=0.1$ and different values of $k_{0} b$. It is seen that the curves of $|R|$ and $|T|$ coincide with those in figure 5 of Porter and Evans [13]. It is observed that $|R|$ decreases and $|T|$ increases as $\alpha$ increases from $0^{\circ}$ to $90^{\circ}$. This is plausible since for a fixed wavenumber, maximum reflection (or minimum transmission) occurs when the waves are normally incident on the barrier.

\section{Conclusion}

Problems of water wave scattering by a submerged vertical barrier extending down to the bottom in uniform finite depth, have been studied here. Galerkin technique involving constant as basis functions multiplied by appropriate weights is used to obtain accurate upper and lower bounds for the reflection and transmission coefficients. This appears to be simple and straightforward compared to other methods employed in the literature to solve this class of problems. Numerical values of upper and lower bounds for the reflection and transmission coefficients coincide within two to three decimal places. Hence, their averages produce very accurate estimates for these coefficients. In the limiting case of deep water and also for uniform finite depth water, graphs of the reflection and transmission coefficients for a submerged barrier extending down to the bottom are recovered as spatial cases. This method can be applied to investigate water wave scattering problems involving barriers of various other geometrical configurations. 


\section{References}

1. Dean WR (1945) On the reflection of surface waves by a submerged plane barrier. Proc Camb Phil 41: 231-238

2. Ursell F (1947) The effect of a fixed barrier on surface wave in deep water. Proc Camb Soc 43: 374-382

3. Havelock TH (1929) Forced surface waves on water. Phil Mag 8: 569-576

4. Williams WE (1966) Note on the scattering of water waves by a vertical barrier. Proc Camb Phil Soc 62: 507-509

5. Evans DV (1970) Diffraction of water waves by a submerged vertical plate. J Fluid Mech 40: 433-451

6. Goswami SK (1982) Scattering of water waves by a submerged fixed vertical barrier in water of finite depth. Bull Cal Math Soc 74: 75-86

7. Goswami SK (1982) Scattering of water waves by a partially immersed fixed vertical barrier in water of finite depth. Bull Cal Math Soc 74: 92-96
8. Goswami SK (1983) Scattering of surface waves by a submerged fixed vertical plate in water of finite depth. J Indian Inst Sci 64: 79-88

9. Smith CM (1983) Some problems in linear water wave theory. Ph.D Thesis, University of Bristol, Bristol

10. Losada IJ, Losada MA, Roldan AJ (1992) Propagation of oblique incident waves past rigid vertical thin barriers. Appl Ocean Res 14: 191-199

11. Mandal BN, Dolai DP (1994) Oblique water wave diffraction by thin vertical barrier in water of uniform finite depth. App Ocean Res 16: 195-203

12. Evans DV, Morris ACN (1972) The effect of a fixed vertical barrier on oblique incident surface waves in deep water. J Inst Maths Applics 9: 198-204

13. Porter R, Evans DV (1995) Complementary approximations to wave scattering by vertical barriers. J Fluid Mech 294: 155-180 